\title{
Seminare/Séminaires/Seminari 2006
}

\section{Praxiseröffnung/-übernahme}

Themen (Details $\rightarrow$ www.fmhservices.ch) Juristische Aspekte - Ehe- und Erbrecht - Praxiseinrichtung - Praxisadministration - Unternehmensbewertung einer Arztpraxis - Finanzierung einer Arztpraxis - Versicherungen.

\section{Sponsoren}

Versicherung der Schweizer Ärzte, Schweizerische Ärzte-Krankenkasse, Druckerei Schmid-Mogelsberg, Labornetz daCapo, Unilabs SA, Labor medica, Kern Concept AG und EMH Schweizerischer Ärzteverlag AG.

\section{Daten}

\begin{tabular}{|c|c|c|}
\hline \multirow[t]{2}{*}{ Nr. 04} & Donnerstag, 7. September 2006 & \multirow[t]{2}{*}{ FMT Zürich } \\
\hline & $9.00-16.30 \mathrm{Uhr}$ & \\
\hline \multirow[t]{2}{*}{ Nr. 05} & Donnerstag, 5. Oktok & \multirow{2}{*}{$\begin{array}{l}\text { Kantons- } \\
\text { spital }\end{array}$} \\
\hline & 16.00-20.30 Uhr & \\
\hline \multirow[t]{2}{*}{ Nr. 06} & Donnerstag, 9. November 2006 & \multirow{2}{*}{$\begin{array}{l}\text { Kantons- } \\
\text { spital }\end{array}$} \\
\hline & 9.00-16.30 Uhr & \\
\hline
\end{tabular}

\section{Praxisübergabe}

\section{Hinweis}

Vor allem aus steuerplanerischer Sicht lohnt es sich, sich bereits frühzeitig (5-10 Jahre) mit diesem Thema auseinanderzusetzen.

\section{Themen (Details $\rightarrow$ www.fmhservices.ch)} Juristische Aspekte - Unternehmensbewertung einer Arztpraxis - Versicherungen - Steuern.

\section{Sponsoren}

Versicherung der Schweizer Ärzte, Schweizerische Ärzte-Krankenkasse, Labor medica und EMH Schweizerischer Ärzteverlag AG.

\section{Daten}

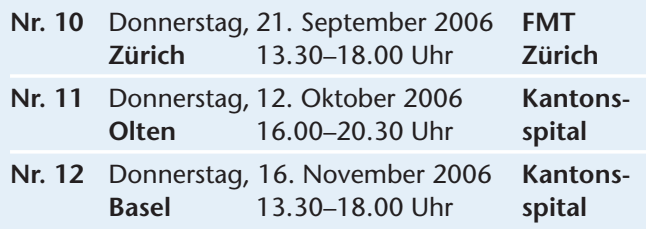

\section{Buchhaltung und Steuern}

Themen (Details $\rightarrow$ www.fmhservices.ch) Buchführung (Massnahmen vor Praxiseröffnung/ -übernahme, Standardkontenplan, doppelte Buchhaltung, EDV-unterstützte Buchführungslösung), Steuern (Steuern als Selbständigerwerbender, Steuerdomizil Privat/Geschäft, strategische und operative Steuerplanung, Steueroptimierungsmöglichkeiten).

\section{Sponsor}

EMH Schweizerischer Ärzteverlag.

\section{Daten}

\begin{tabular}{|c|c|c|c|}
\hline Nr. 15 & Donnerstag, & 14. September 2006 & FMT \\
\hline & Zürich & 9.30-16.30 Uhr & Zürich \\
\hline Nr. 16 & $\begin{array}{l}\text { Donnerstag, } \\
\text { Basel }\end{array}$ & $\begin{array}{l}\text { 23. November } 2006 \\
9.30-16.30 \mathrm{Uhr}\end{array}$ & $\begin{array}{l}\text { Kantons- } \\
\text { spital }\end{array}$ \\
\hline
\end{tabular}

\section{Praxiscomputerworkshop}

\section{Inhalt (Details $\rightarrow$ www.fmhservices.ch)}

Präsentation von sechs Praxisadministrationssoftwarelösungen (Leistungserfassung, elektronisches Abrechnen unter Einbezug der TrustCenter, Agendaführung, elektronische Krankengeschichte, Statistiken, Laborgeräteeinbindung, Finanzbuchhaltungslösungen usw.).

\section{Ziel}

Die Workshopteilnehmer/innen erhalten einen ersten Überblick über führende Softwarelösungen. Sie erfahren, wie bei der Evaluation eines Ärztepaketes zweckmässigerweise vorgegangen wird und welche Fehler vermieden werden sollten.

\section{Datum}

Nr. 19 Donnerstag, 30. November 2006 Hotel InterZürich 14.00-17.30 Uhr continental

\section{Röntgen in der Arztpraxis}

Themen (Details unter www.fmhservices.ch) Vom konventionellen zum digitalen Röntgen Rentabilität Röntgen in der Arztpraxis - Neue Vorschriften ab 1. Januar 2008 - Evaluation und Beschaffung neuer oder gebrauchter Anlagen Komplette Marktübersicht mit Preisen und Leistungskomponenten. 


\section{Kosten}

Fr. 300.- (inkl. sämtlicher Kursunterlagen und Verpflegung)

\section{Daten}

\begin{tabular}{|c|c|c|c|}
\hline Nr. 21 & $\begin{array}{l}\text { Donnerstag, } \\
\text { Burqdorf }\end{array}$ & $\begin{array}{l}\text { 7. September } 2006 \\
9.30-16.00 \text { Uhr }\end{array}$ & $\begin{array}{l}\text { Typon } \\
\text { Imaqing AG }\end{array}$ \\
\hline r. 22 & Dor & 9. Nove & Idungs- \\
\hline & Cha & $9.30-16$ & $\begin{array}{l}\text { zentrum } \\
\text { Galexis }\end{array}$ \\
\hline
\end{tabular}

\section{Ouverture et gestion d'un cabinet médical}

\section{Contenu (Détails $\rightarrow$ www.fmhservices.ch)}

Aménagement - Financement - Administration d'un cabinet médical - Passage du statut de salarié à celui d'indépendant - Assurances - Aspects juridiques et fiscaux - News de l'AMG/SVMED Laboratoire - Atelier questions.

\section{Sponsors}

Assurance des Médecins Suisses, Caisse-Maladie des Médecins Suisses, Unilabs SA et EMH Editions médicales suisses SA.

\section{Date}

$\begin{array}{lll}\text { № } 29 & \text { Jeudi } 28 \text { septembre } 2006 & \text { HUG } \\ & \text { Genève } 9.00-17.00 \mathrm{~h} & \end{array}$

\section{Remise/reprise d'un cabinet médical}

\section{Contenu (Détails $\rightarrow$ www.fmhservices.ch)}

Aspects juridiques - Droit matrimonial et successoral - Mesures architecturales - Assurances - Estimation d'inventaire et goodwill d'un cabinet Conséquences fiscales d'une remise/reprise News de l'AMG/SVMED - Laboratoire - Atelier questions.

\section{Sponsors}

Assurance des Médecins Suisses, Caisse-Maladie des Médecins Suisses et EMH Editions médicales suisses SA.

\section{Date}

$\begin{array}{lll}\text { NNo } 31 & \text { Jeudi } 16 \text { novembre } 2006 & \text { HUG } \\ \text { Genève } & 9.00-16.30 \mathrm{~h} & \end{array}$

\section{Contabilità e fiscalità (Ticino)}

L'obiettivo di questo seminario è di dare al medico le conoscenze necessarie per utilizzare autonomamente lo strumento contabile e per operare con più efficienza ed economicità, nonché far comprendere al medico il significato della pianificazione fiscale.
Mediante esempi pratici elaborati in gruppo, verrà illustrato in maniera semplice ma completa il sistema di gestione della contabilità di uno studio medico con effetto anche sulla pianificazione fiscale.

\section{Data \\ № 34 Giovedì 19 ottobre $2006 \quad$ FMH Fiduciaria Chiasso dalle 9.00 alle 16.00 Services}

\section{Anmeldung und Auskunft / Inscription et information}

FMH Consulting Services, Claudine Tannari, Burghöhe 1, 6208 Oberkirch, Tel. 04192500 77, Fax 0419210586 oder via Internet unter www.fmhservices.ch.

\section{Hinweis/Remarque}

Bei sämtlichen Seminaren, bei denen die Kosten teilweise oder gänzlich von Seminarsponsoren gedeckt werden, werden die Teilnehmeradressen den jeweiligen Sponsoren zur Verfügung gestellt. Les adresses des participants aux séminaires dont les coûts sont couverts en partie ou totalement par des sponsors sont communiquées aux sponsors concernés.

\section{Annullierungsbedingungen/ \\ Conditions d'annulation}

Bei den Gratisseminaren werden bei Abmeldungen oder Fernbleiben folgende Unkostenbeiträge erhoben:

Pour les séminaires gratuits, un montant est perçu pour absence ou annulation. Il est de:

- Fr. 50.- pro Person ab 14 Tage vor Seminarbeginn / par personne dans les 15 jours avant;

- Fr. 80.- pro Person ab 7 Tage vor Seminarbeginn oder bei Fernbleiben/par personne dans les 7 jours avant le début du séminaire. Bei Abmeldungen oder Fernbleiben bei kostenpflichtigen Seminaren werden die folgenden Unkostenbeiträge erhoben:

Pour les séminaires payants, un montant est perçu pour une absence ou une annulation. Il est de:

- 30\% der Seminarkosten ab 14 Tage vor Seminarbeginn / des coûts du séminaire dans les 15 jours avant le début du séminaire;

- 100\% der Seminarkosten ab 7 Tage vor Seminarbeginn oder bei Fernbleiben/des coûts du séminaire dans le 7 jours avant le début du séminaire. 